\title{
Intercomparaciones a nivel de radioprotección con cámara de ionización de transferencia en la calidad de radiación ${ }^{137} \mathrm{Cs}$
}

\section{Interlaboratory comparisons at protection level with transfer ionization chamber using radiation quality ${ }^{137} \mathrm{Cs}$}

Camilo Ernesto Calderón ${ }^{1}$, Laura Quintero ${ }^{1}$, Wilson Eduardo Moreno ${ }^{1}$, Julián Andrés Niño ${ }^{1}$

Citación: C. E. Calderón, L. Quintero, W. E. Moreno y J. A. Niño, "Intercomparaciones a nivel de radioprotección con cámara de ionización de transferencia en la calidad de radiación ${ }^{137} \mathrm{Cs}$ ", Revista de Investigaciones y Aplicaciones Nucleares, n. ${ }^{\circ}$ 2, pp. 55-61, 2018.

Recibido: 11 de julio de 2018

Aceptado: 22 de octubre de 2018

Publicado en línea: 17 de

diciembre de 2018

Doi: 10.32685/2590-7468/invapnuclear.2.2018.59

Palabras claves: Intercomparación, radioprotección, trazabilidad.

Keywords:

Intercomparison, radioprotection, traceability.

\section{RESUMEN}

En el presente trabajo se exponen la metodología y los resultados obtenidos de los ejercicios de intercomparación que fueron adelantados por el Laboratorio Secundario de Calibración Dosimétrica (LSCD) del Servicio Geológico Colombiano en los años 2016 y 2017, con el Laboratorio Secundario del Centro de Protección e Higiene de las Radiaciones (CPHR), de Cuba, y el Laboratorio de Dosimetría del Organismo Internacional de Energía Atómica (OIEA), mediante la calibración de cámaras de transferencia en la magnitud tasa de kerma en aire con haz de ${ }^{137} \mathrm{Cs}$ por el método que usualmente sigue cada laboratorio. De dichos ejercicios se obtuvieron resultados satisfactorios con respecto a la metodología de calibración normalizada que es implementada en el LSCD, la cual a su vez brinda confiabilidad en sus resultados y en la trazabilidad de las magnitudes operacionales en las medidas de campo de los instrumentos utilizados en protección radiológica en Colombia.

\section{ABSTRACT}

This paper presents the methodology used and the results obtained for the inter-laboratory comparisons performed by the Secondary Standards Dosimetry Laboratory (LSCD) from the Servicio Geológico Colombiano (SGC) in 2016 and 2017. The comparisons were performed with the Cuban LSCD of Centro de Protección e Higiene de las Radiaciones (CPHR), and the Dosimetry Laboratory of the International Atomic Energy Agency (IAEA), by means of the calibration of transfer ionization chambers in terms of the quantity air Kerma with a ${ }^{137} \mathrm{Cs}$ beam. Each laboratory performed the calibration following their own established procedure. The results were satisfactory for both comparisons, ensuring the traceability and reliability of the calibration of instruments used with radiological protection purposes all around the country.

${ }^{1}$ Dirección de Asuntos Nucleares, Servicio Geológico Colombiano

Email de correspondencia: ccalderon@sgc.gov.co 


\section{INTRODUCCIÓN}

D e acuerdo con las propiedades inherentes de las radiaciones ionizantes y sus efectos asociados (efectos deterministas y efectos estocásticos), y la necesidad de cuantificar el posible daño biológico que pueden causar, se han establecido magnitudes limitadoras, como la dosis equivalente en órgano y la dosis efectiva, pero para efectos prácticos, con el objeto de tener una medida razonable y sobrestimada de las magnitudes limitadoras, se propusieron las llamadas magnitudes operacionales, como la dosis equivalente ambiental y la dosis equivalente personal, que son medibles en los equipos de campo y en equipos de dosimetría personal con fines de protección radiológica. Lo anterior hace necesario implementar, mantener y desarrollar patrones de referencia que permitan brindar una trazabilidad confiable relacionada con las mediciones que se hacen en áreas como la medicina, en la cual se requiere que sean lo más exactas posible las dosis administradas por los haces de radiación en terapias de tumores de pacientes con cáncer, y en la industria e investigación científica; más aún, es menester que el método de calibración en el que se basa la trazabilidad de las magnitudes operacionales sea confiable.

Por tal motivo, es de gran importancia para el Laboratorio Secundario de Calibración Dosimétrica (LSCD) del Servicio Geológico Colombiano (SGC), como referente en metrología de las radiaciones ionizantes en el ámbito nacional, buscar, mediante las intercomparaciones internacionales, resultados satisfactorios para así proporcionar confianza a los usuarios, lo cual contribuye a brindar mediciones de radiación ionizante fiables y trazables. Igualmente, entre los principales objetivos del OIEA se encuentra el de apoyar a los países en la utilización de técnicas nucleares e isotópicas [1]; por ello, el OIEA estima necesaria la verificación dosimétrica, para que los laboratorios de calibración dosimétrica demuestren su competencia y de esta manera se puedan mantener adecuadamente las capacidades de medición de radiación ionizante.

Para mantener la confianza en la cadena de trazabilidad, el OIEA recomienda que los los laboratorios secundarios que brindan servicio de calibración participen en programas de comparación al menos cada cinco años, o cuando hayan cambiado, sus estándares de referencia, configura- ciones de irradiación o la técnica de medición [2]. En lo concerniente a comparaciones realizadas a nivel de radioprotección, el objetivo del programa dirigido por el OIEA es verificar que los laboratorios secundarios pueden prestar el servicio de calibración en términos de las magnitudes operacionales establecidas por la Comisión Internacional de Unidades Radiológicas (ICRU), dentro de los límites aceptables en las diferentes calidades de radiación. Como parte de estos programas se envían cámaras calibradas de ionización de transferencia del OIEA a los diferentes laboratorios participantes con el objeto de que las calibren mediante su propio procedimiento [3].

El objetivo del presente trabajo es dar a conocer los resultados de dos intercomparaciones en magnitud tasa de kerma en aire con cámara de trasferencia, en la cuales participó el LSCD y obtuvo resultados satisfactorios en ambas comparaciones. El fin es mostrar la competencia que tiene el LSCD en la confiabilidad de la trazabilidad de las magnitudes operacionales como la dosis equivalente ambiental $H^{\star}(10)$ y la dosis equivalente personal $H_{\mathrm{p}}(10)$. Estas magnitudes son calculadas a partir de la tasa de kerma en aire de referencia, que es una magnitud dosimétrica fundamental. Las intercomparaciones fueron realizadas en primera instancia en el Laboratorio Secundario del Centro de Protección e Higiene de las Radiaciones (CPHR), de Cuba, en 2016, y luego en el Laboratorio de Dosimetría del OIEA, en 2017.

\section{MATERIALES Y MÉTODOS}

\subsection{Condiciones de calibración en haces de ${ }^{137} \mathrm{Cs}$ usadas en la intercomparación en el CPHR}

En la tabla 1 se muestran los parámetros que se tuvieron en cuenta para calibrar la cámara de transferencia enviada por el CPHR, tales como la magnitud utilizada, el tipo de irradiador, radionucleido, tamaño de campo y la tasa de kerma en aire de referencia.

Los tamaños de campo usados para realizar las mediciones son distintos debido a que los colimadores de cada uno de los irradiadores son de diferente diámetro; sin embargo, esta diferencia no influye en el resultado de las mediciones, pues la cámara es irradiada en su totalidad, ya que el diámetro aproximado de la cámara de transferencia es de $14 \mathrm{~cm}$, y ambos tamaños de campo cubren totalmente este diámetro. 
Tabla 1. Condiciones de calibración para intercomparación en el CPHR

\begin{tabular}{|c|c|c|c|c|c|c|}
\hline Magnitud dosimétrica & Institución & Irradiador & Radionucleido & $\begin{array}{c}\text { Tamaño del campo } \\
\text { (cm) }\end{array}$ & $\mathrm{DFC} *(\mathrm{~cm})$ & $\begin{array}{c}\text { Tasa de kerma en aire } \\
\quad * *(\mathrm{mGy} / \mathrm{h})\end{array}$ \\
\hline & CPHR & OB6 & ${ }^{137} \mathrm{Cs}$ & $\emptyset=75$ & 300 & 3,06 \\
\hline kerma en aire & LSCD & $\begin{array}{l}\text { Hopewwll Designs, } \\
\text { INC G10-1-12-20 LS }\end{array}$ & ${ }^{137} \mathrm{Cs}$ & $\varnothing=90$ & 300 & 5,63 \\
\hline
\end{tabular}

* Distancia entre la fuente y la cámara de ionización.

** Tasa de kerma en aire durante la calibración.

Fuente: autores

\subsubsection{Sistemas dosimétricos de referencia}

La tabla 2 compila la información sobre los sistemas dosimétricos utilizados y la trazabilidad de estos, procedimiento realizado por los dos laboratorios para el proceso de intercomparación.

En la figura 1 se muestran el irradiador G-10 del LSCD y el sistema dosimétrico de referencia implementado en el proceso.

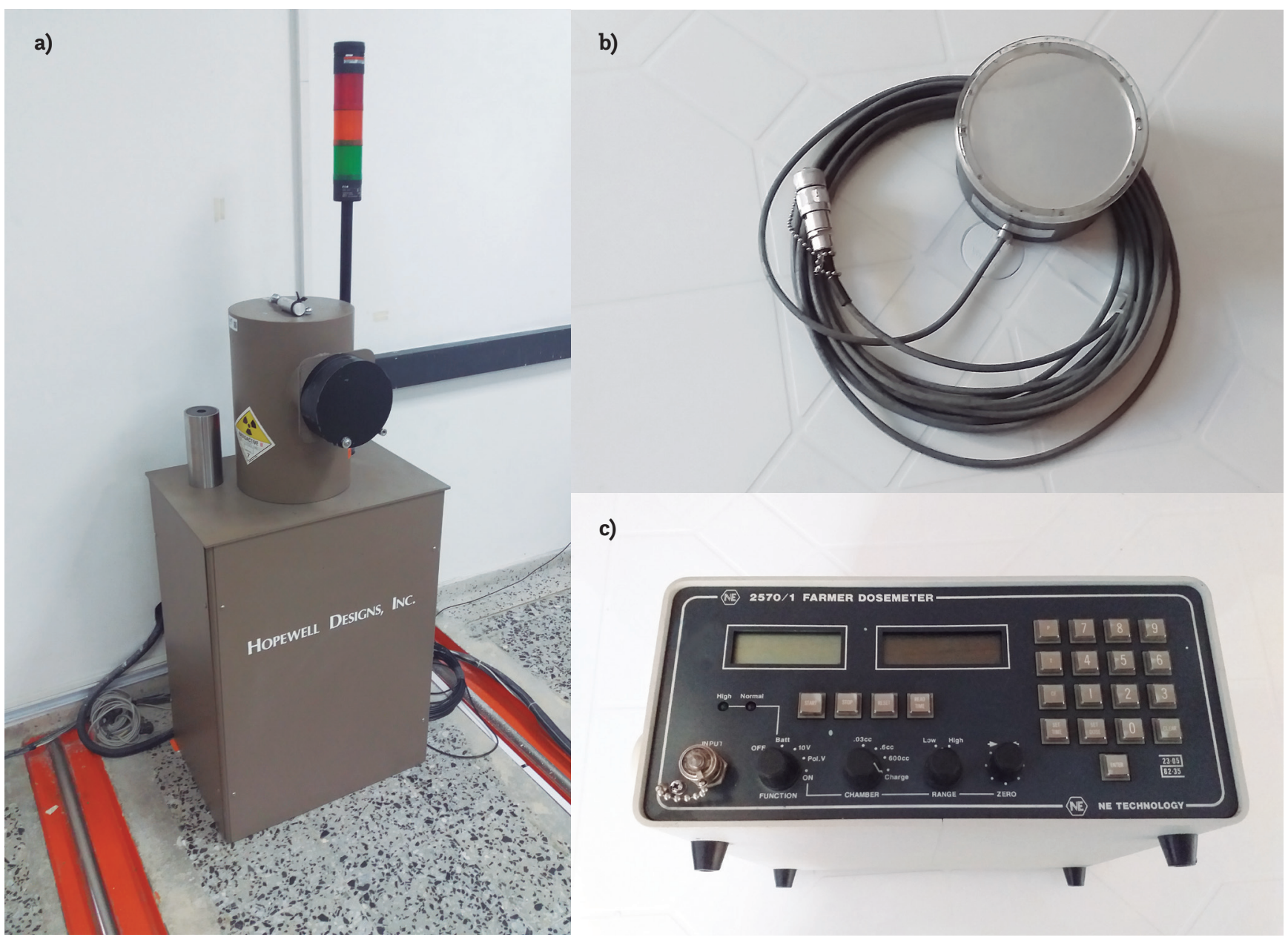

Tabla 2. Sistemas dosimétricos de referencia y su trazabilidad

\begin{tabular}{ccc} 
& CPHR & LSCD \\
\cline { 2 - 3 } Cámara de ionización & PTW 32002 N/S 073 & NE 2575 N/S 136 \\
Trazabilidad & OIEA/BIPM, 2016 & OIEA/BIPM, 2014 \\
Electrómetro & Unidos Webline S/N 023 & NE 2570 N/S 1104 \\
\hline
\end{tabular}

Fuente: autores

Figura 1. a) Irradiador G-10-1-12-20 Cs. b) Cámara patrón de referencia Farmer 2575. c) Electrómetro Farmer 2570. Fuente: autores 


\subsubsection{Instrumentos utilizados en la intercomparación bilateral realizada en el Laboratorio del OIEA}

En la tabla 3 se muestran los parámetros tenidos en cuenta para la calibración de la cámara de transferencia enviada por el OIEA, como magnitud, radionucleido y la tasa de kerma en aire de referencia.

Las características técnicas de la cámara de transferencia enviada por el Laboratorio de Dosimetría del OIEA (figura 2) se encuentran en la tabla 4.
Para la intercomparación con los resultados del OIEA se utilizó el mismo sistema dosimétrico de referencia utilizado por el LSCD, mostrado en las figuras 1b, 1c, y para la calibración de la cámara de trasferencia remitida por el OIEA se utilizó el electrómetro modelo PTW Unidos E, serie 900092. El OIEA no reportó el tipo de electrómetro con el que realizó la calibración de su cámara de trasferencia.

Tabla 3. Condiciones de calibración para intercomparación realizada en el laboratorio del OIEA

\begin{tabular}{cccccc}
\hline Magnitud dosimétrica & Institución & Radionucleido & DFC* $(\mathrm{cm})$ & $\begin{array}{c}\text { Tasa de kerma en aire** } \\
(\mathrm{mGy} / \mathrm{h})\end{array}$ & \multicolumn{1}{c}{ Trazabilidad } \\
\hline \multirow{2}{*}{ Kerma en aire } & OIEA & ${ }^{137} \mathrm{Cs}$ & 300 & 3,60 & BIPM \\
& LSCD & ${ }^{137} \mathrm{Cs}$ & 300 & 5,49 & OIEA \\
\hline
\end{tabular}

** Tasa de kerma en aire durante la calibración.

Fuente: autores

Tabla 4. Características de la cámara de transferencia utilizada en la intercomparación en el laboratorio del OIEA

\begin{tabular}{ccccc}
\hline Modelo & Punto de referencia & Volumen nominal (cm $\left.)^{3}\right)$ & Voltaje de polarización $(\mathrm{V})$ & $\begin{array}{c}\text { Espesor de las paredes } \\
(\mathbf{m m})\end{array}$ \\
\hline Exradin A6 & Centro de la cámara & 800 & 400 & 3,0 \\
\hline
\end{tabular}

Fuente: autores

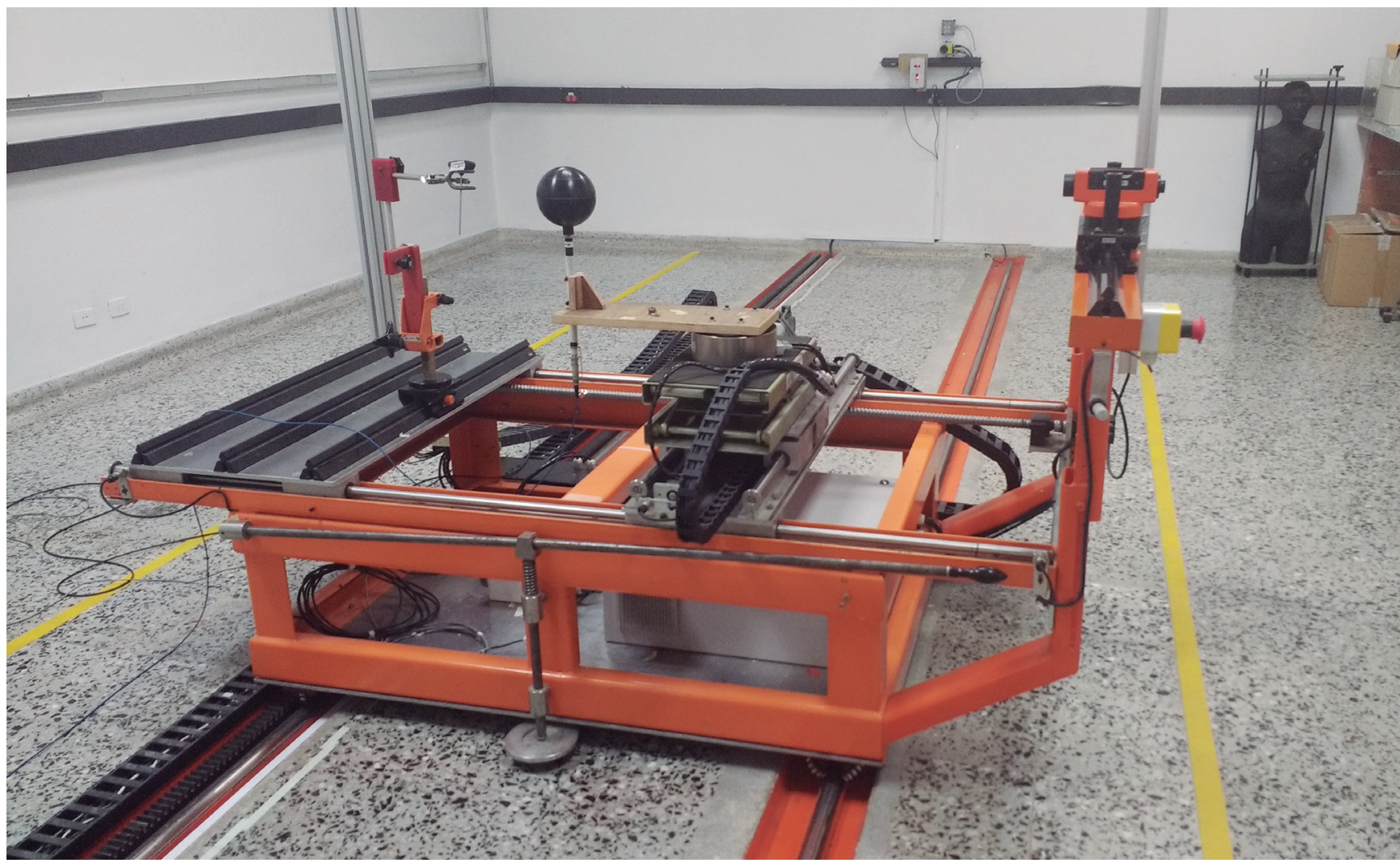

Figura 2. Cámara de transferencia utilizada en la intercomapración en el laboratorio del OIEA. Fuente: autores 


\subsection{Método}

\subsubsection{Cálculo del factor de calibración de la cámara de transferencia}

La metodología implementada con el fin de calcular el factor de calibración de la cámara de transferencia fue la misma en los dos procesos de intercomparación realizados por el LSCD. El procedimiento general se describe a continuación:

En primer lugar, se realiza una prueba de fugas con el fin de determinar la corriente promedio registrada por el electrómetro cuando la cámara de ionización no se encuentra expuesta a la radiación. El valor de la corriente de fugas se debe tener en cuenta durante la estimación de la incertidumbre; este valor no debe superar el $2 \%$ de la corriente que se obtiene al exponer la cámara de ionización a la radiación.

Con la cámara de referencia posicionada a una distancia de 3 m (figura 3) de la fuente se calculó directamente la tasa de kerma en aire de referencia. Se realizaron seis series, cada una de diez mediciones de carga $Q$, con un tiempo de colección $t$ de 240 segundos, con lo cual se obtiene la corriente de ionización $I$. Cada vez que se realizaron dos mediciones consecutivas, y al final de la serie, se registró el valor de las condiciones ambientales para calcular el factor de corrección por desviaciones de presión atmosférica y temperatura respecto a las condiciones de referencia $C_{\mathrm{PT}}$ y corregir las medidas de corriente $I_{\text {corr }}=I C_{\mathrm{PT}}$. Con dichas mediciones se puede calcular la tasa de kerma en aire $\left(\dot{K}_{\text {ref }}\right)$ por medio de la siguiente ecuación:

$$
\dot{K}_{\text {ref }}=N_{\text {ref }} I_{\text {corr }}
$$

\section{Donde}

$N_{\text {ref }}$ es el factor de calibración de la cámara de referencia; $I_{\text {corr }}$ es la corriente promedio corregida por el factor $C_{\mathrm{PT}}$ de todas las mediciones.

Luego se posicionó la cámara de transferencia a $3 \mathrm{~m}$, se le realizó una prueba de fugas y se realizaron cuarenta mediciones de corriente de ionización corregida por el factor presión-temperatura $I_{\text {tras }}$, con un tiempo de irradiación de 30 segundos. El factor de calibración de la cámara se calcula mediante la siguiente ecuación:

$$
N_{\text {tran }}=\frac{\dot{K}_{\text {ref }}}{I_{\text {tras }}}
$$

Donde

$\dot{K}_{\text {ref }}$ es la tasa de kerma en aire de referencia calculada con la ecuación (1).

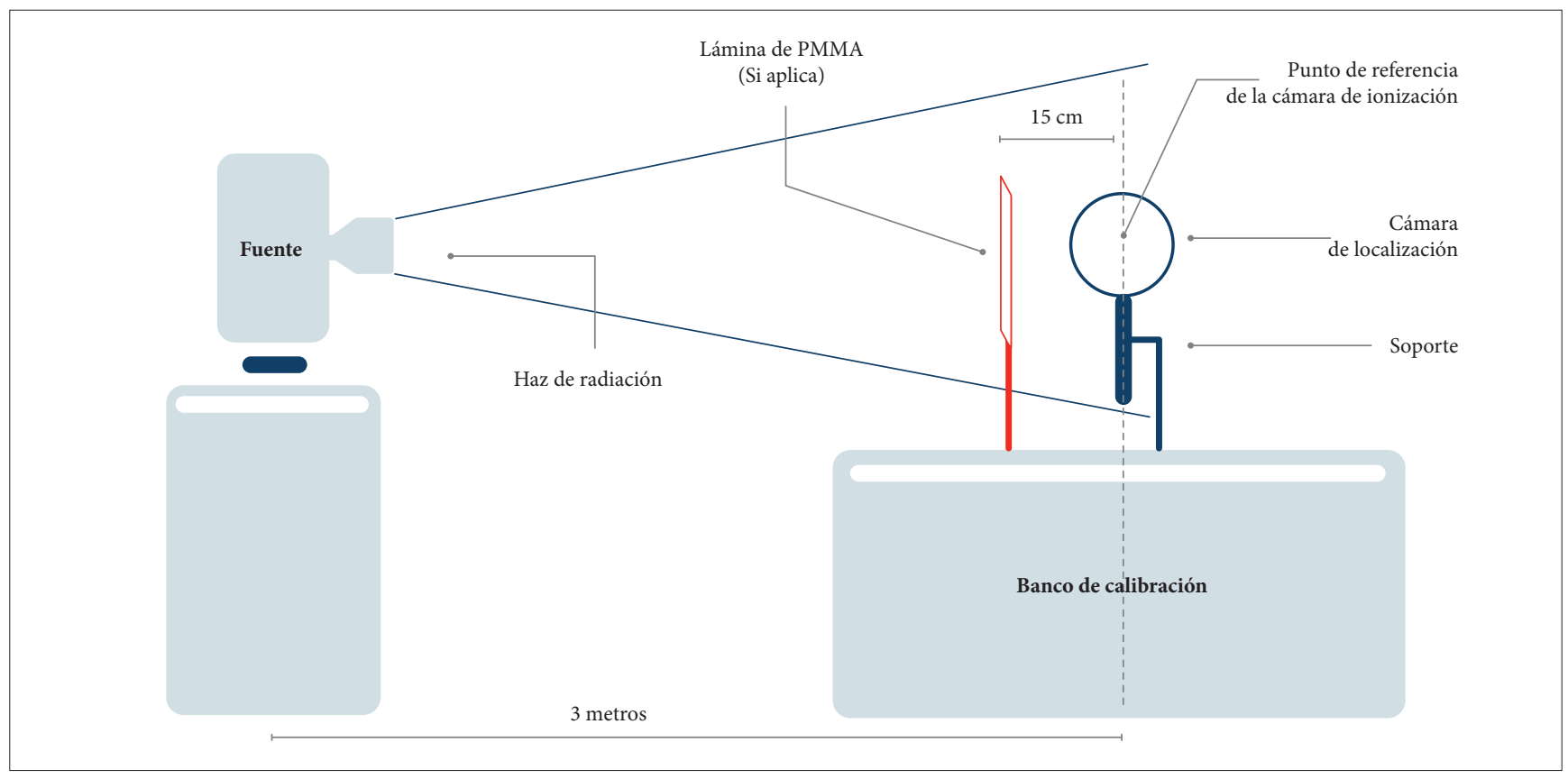

Figura 3. Montaje experimental utilizado en las mediciones, en las intercomparaciones. 


\subsubsection{Cálculo de incertidumbre}

En la tabla 5 se muestran las fuentes de incertidumbres tenidas en cuenta por LSCD en las mediciones con la cámara patrón de referencia para el cálculo de la tasa de kerma en aire de referencia para cada una de las intercomparaciones

Tabla 5. Incertidumbre reportada por el LSCD en el cálculo de la tasa de kerma en aire de referencia

\begin{tabular}{lcc}
\hline Fuente de incertidumbre & $\begin{array}{c}\text { Incertidumbre }(\%) \mathbf{k}=\mathbf{1} \text { reportada por el LSCD en la } \\
\text { intercomparación realizada en los dos laboratorios } \\
\text { CPHR }\end{array}$ & OIEA \\
\hline Calibración del patrón & 0,41 & 0,41 \\
Condiciones ambientales & 0,28 & 0,13 \\
Posicionamiento & 0,01 & 0,05 \\
Estabilidad del patrón & 0,94 & 0,94 \\
Homogeneidad del haz & 0,58 & 0,58 \\
Rotación e inclinación de & 0,29 & 0,29 \\
la cámara & 0,05 & 0,06 \\
Mediciones con el patrón & $1,20 \%$ & $1,22 \%$ \\
Ukair & & \\
\hline
\end{tabular}

* Incertidumbre expandida de la tasa de kerma en aire.

Fuente: autores

En la tabla 6 se muestran las incertidumbres en la calibración del instrumento de trasferencia reportadas por el LSCD.

Tabla 6. Incertidumbre reportada por el LSCD en el cálculo del factor de calibración con las cámaras de transferencia en cada intercomparación

\begin{tabular}{|c|c|c|}
\hline \multirow{2}{*}{ Fuente de incertidumbre } & \multicolumn{2}{|c|}{$\begin{array}{l}\text { Incertidumbre }(\%) k=1 \text { reportada por el LSCD en la } \\
\text { intercomparación realizada en los dos laboratorios }\end{array}$} \\
\hline & CPHR & OIEA \\
\hline Tasa de Kair de referencia & 1,20 & 1,22 \\
\hline Condiciones ambientales & 0,15 & 0,11 \\
\hline Posicionamiento & 0,06 & 0,05 \\
\hline $\begin{array}{l}\text { Corrección por el uso de } \\
\text { un electrómetro diferente }\end{array}$ & 0,58 & 0,19 \\
\hline Homogeneidad del haz & 0,58 & 0,58 \\
\hline $\begin{array}{l}\text { Linealidad del } \\
\text { electrómetro }\end{array}$ & 0,29 & 0,29 \\
\hline $\begin{array}{l}\text { Mediciones con la cámara } \\
\text { de transferencia }\end{array}$ & 0,04 & 0,06 \\
\hline Ukair* & $1,50 \%$ & $1,40 \%$ \\
\hline
\end{tabular}

* Incertidumbre expandida de la tasa de kerma en aire.

Fuente: los autores

\subsubsection{Método estadístico}

\subsubsection{Intercomparación con el CPHR}

Para el análisis de los resultados obtenidos en la intercomparación en el CHPR se utilizó el estadígrafo recomendado en la ISO/IEC $17043[7,8]$

$$
E_{n}=\frac{x-X}{\sqrt{U_{\mathrm{lab}}^{2}-U_{\text {ref }}^{2}}}
$$

Donde

$x$ es el valor del factor de calibración dado por el participante;

$X$ es el valor del factor de calibración dado por el laboratorio de referencia;

$U_{\text {lab }}$ es la incertidumbre expandida del resultado del participante;

$U_{\text {ref }}$ es la incertidumbre expandida del valor asignado por el laboratorio de referencia.

Para los casos,

$E_{n} \leq 1,0$ indica desempeño satisfactorio;

$E_{n} \geq 1,0$ indica desempeño insatisfactorio.

\subsubsection{Intercomparación en el laboratorio del OIEA}

En este caso, el método estadístico utilizado fue el sugerido por el protocolo de intercomparación del OIEA [1], en el cual el resultado de la comparación puede ser evaluado mediante la siguiente relación:

$$
R=\frac{N_{\text {part }}}{N_{\text {ref }}}
$$

Donde

$N_{\text {part }}$ es el coeficiente de calibración determinado por el participante;

$N_{\text {ref }}$ es el coeficiente de calibración tomado como referencia.

El resultado de comparación $R$ se considera aceptable si es consistente (la incertidumbre expandida de $R$ cubre el valor unitario) y si el valor está entre $0,97 \leq R \leq 1,03$. 


\section{RESULTADOS Y DISCUSIÓN}

\subsection{Intercomparación bilateral con el CPHR}

En la tabla 7 se muestran los resultados de los coeficientes de calibración y su respectiva incertidumbre en la cámara de trasferencia obtenidos por el CPHR y el LSCD, así como el valor del estadígrafo y la incertidumbre de la intercomparación.

Tabla 7. Resultados de la intercomparación en el CPHR

\begin{tabular}{ccccc}
\hline Participante & $N_{\text {trans }}(\mu \mathrm{Gy} / \mathrm{nC})$ & $\begin{array}{c}\text { Incertidumbre } \\
(\%) k=1\end{array}$ & $E_{n}$ & $U_{r}(\%)$ \\
\hline CPHR & 24,99 & 0,96 & 0,1 & 3,56 \\
LSCD & 25,04 & 1,5 & & \\
\hline
\end{tabular}

$U_{\mathrm{r}}(\%)$; es la incertidumbre expandida $(k=2)$ de la intercomparación $U_{r}=\sqrt{U_{\mathrm{LSCD}}^{2}+U_{\mathrm{CPHR}}^{2}}$

Fuente: autores

\subsection{Intercomparación con el Laboratorio}

\section{de Dosimetría del OIEA}

En la tabla 8 se muestran los resultados de los coeficientes de calibración y su respectiva incertidumbre para la cámara de trasferencia obtenidos por el OIEA y el LSCD, el valor del estadígrafo y la incertidumbre

Tabla 8. Resultados de la intercomparación con el OIEA

\begin{tabular}{ccccc}
\hline Participante & $N_{\text {trans }}(\mu \mathrm{Gy} / \mathrm{nC})$ & $\begin{array}{c}\text { Incertidumbre } \\
(\mu \mathrm{Gy} / \mathrm{nC}) k=2\end{array}$ & $R$ & $U_{r}(\%)$ \\
\hline OIEA & $(37,769)$ & 0,30 & 1,003 & 2,9 \\
LSCD & $(37,865)$ & 1,06 & & \\
\hline
\end{tabular}

$U_{r}(\%)$ es la incertidumbre expandida $(k=2)$ de la intercomparación. Fuente: autores

Los resultados obtenidos en los ejercicios de intercomparación permitieron confirmar metrológicamente la metodología de calibración normalizada que es implementada en el LSCD, lo cual hace confiables las actividades de prestación de servicio a los usuarios y contribuye a brindar una trazabilidad fiable al realizar las mediciones de radiación ionizante. Dado que en las pruebas realizadas el valor de $E_{n}$ se encuentra en los rangos satisfactorios, los resultados del ejercicio realizado en el CPHR también se consideran, en general, satisfactorios. De esta manera el LSCD ha demostrado que la prestación de servicio de calibración, en términos de magnitudes operacionales establecidas por la Comisión Interna- cional de Unidades Radiológicas, se encuentra en los límites aceptables, ya que el valor del estadígrafo $R$ se encuentra en el rango de $0,97 \leq R \leq 1,03$. Esto significa que se han logrado mantener las capacidades de medición de radiación ionizante.

Finalmente, los procesos de intercomparación adelantados por el LSDC y los resultados obtenidos constituyen un incentivo para el grupo de trabajo, cuyo propósito es seguir participando en dichos procesos para lograr una mayor confiabilidad y desarrollar nuevas metodologías de calibración.

\section{Referencias}

[1] International Atomic Energy Agency, Technical Protocol of the Radiation Protection Comparison.

[2] Requisitos generales para la competencia de los laboratorios de ensayo y de calibración. ISO 17025.2005. [En línea]. Disponible en https://www.iso.org/obp/ui/\#iso:std:iso-iec:17025:ed-2:v1:es

[3] Carta de la Red de Laboratorios Secundarios de Calibración Dosimetrica (SSDL), 2. ${ }^{\text {a }}$ ed., Viena: Organismo Internacional de Energía Atómica, 2018.

[4] Appendix of the IAEA Calibration Certificate: Calibration of Reference Dosimeters for Diagnostic Radiology at the IAEA Dosimetry Laboratory. [En línea]. Disponible en http://www-naweb.iaea.org/nahu/dmrp/documents/DOLP_011_Appendix_3B.pdf

[5] X and Gamma Reference Radiation for Calibrating Dosemeters and Doserate Meters and for Determining their Response as a Function of Photon Energy, Ginebra: ISO 4037, 2004.

[6] International Atomic Energy Agency, "Calibration of Radiation Protection Monitoring Instruments", IAEA Safety Reports Series, n. ${ }^{\circ}$ 16, Viena: IAEA, 2000.

[7] Norma cubana, "Evaluación de la conformidad: requisitos generales para los ensayos de aptitud", La Habana: NC-ISO/IEC 17043, 2011.

[8] G. Walwyn-Salas, L. Czap, I. Gomola, J. A. Tamayo-García, “Testing the Performance of Dosimetry Measurement Standards for Calibrating Area and Personnel Dosimeters", Journal of Instrumentation, vol. 11, n. ${ }^{\circ} 7$, P07005-P07005, 2016. 